\title{
NUMERICAL STRESS ANALYSIS FOR PREDICTION OF CRACK PROPAGATION DIRECTION IN ADHESIVE LAYER
}

\author{
$\underline{\text { N.J. Al-Ramahi }}{ }^{1,2}$, R. Joffe ${ }^{1 *}$, J. Varna $^{1}$ \\ ${ }^{1}$ Luleå University of Technology, Luleå, Sweden \\ ${ }^{2}$ Middle Technical University, Baghdad, Iraq \\ *Roberts.Joffer@1tu.se
}

The study outlined here is related to the prediction of crack propagation path in the adhesive layer of joints. Typically crack initiation is predicted from stress analysis while fracture mechanics deals with propagation [1]. The fracture mechanics for complex stress states does not have an analytical solutions and numerical simulation should be employed. Besides, the crack propagation occurs in small increments with changing directions and more than one numerical simulation may be needed for each step and fracture toughness in a form of critical Energy Release Rate (ERR) for different propagation modes is needed as input. Thus, the procedure becomes rather time and resource consuming. The current paper offers alternative methodology which is based on the analysis of stress state ahead of existing crack to predict direction of the crack growth. The double cantilever beam with similar/dissimilar materials and adhesive layer in-between them is chosen as an example and the stress analysis is performed by means of finite element method. It is shown that crack path may be predicted based on the maximum peel (hoop) stress found on the circle of fixed radius around the crack tip. The crack extension is modeled in incremental manner with step-by-step procedure. Only two increments from the initial crack in the adhesive layer are considered. The angle $\theta_{l}$ between the pre-crack and maximum value of the peel stress defines direction for the first crack increment. The direction for the second increment is defined by angle $\theta_{2}$ with respect to the first increment. Thus, two angles are identified: $\theta_{1}$ - deviation from the horizontal pre-crack; $\theta_{2}$ - second increment (see Figure 1a). The results are verified by traditional fracture mechanics approach to calculate work needed to close the crack increment (see Figure 1b).
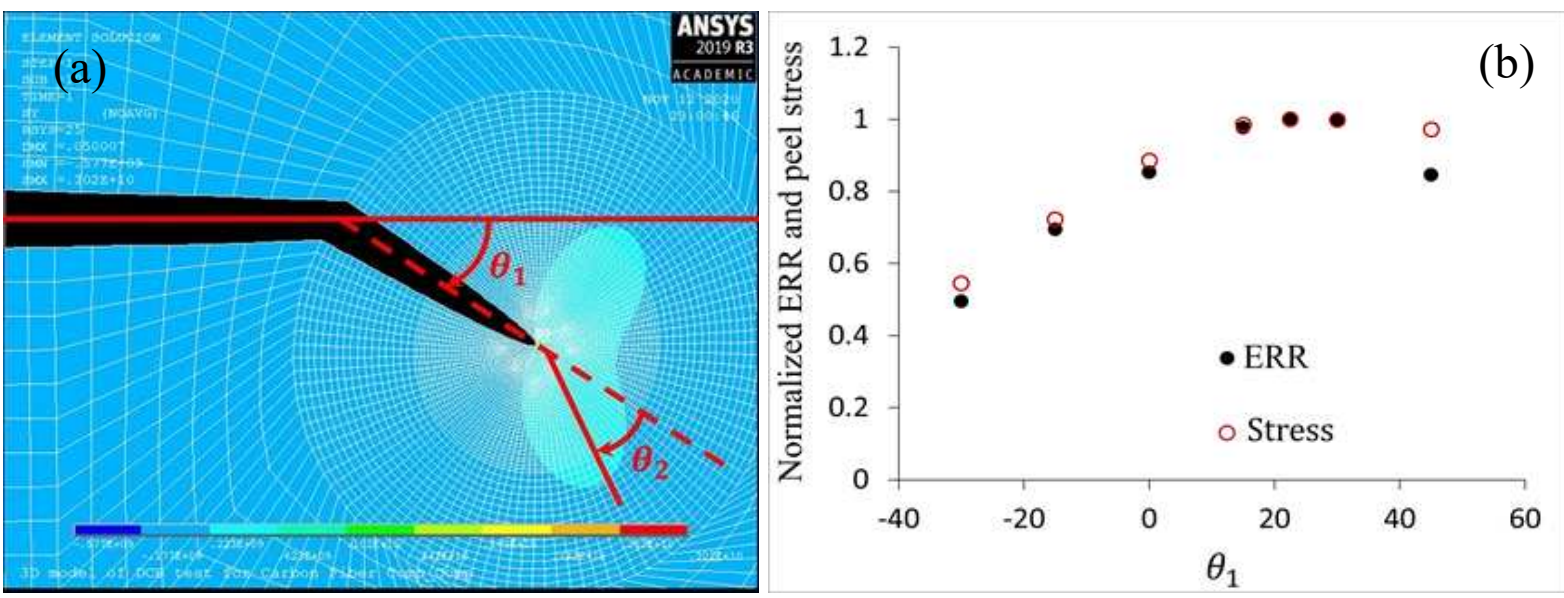

Figure 1. Angles defining crack propagation direction (a) and Comparison between peel (hoop) stress distribution and energy release rate (ERR) for different possible angles of crack extension.

\section{References}

[1] J.A.G. Chousal, M.F.S.F. de Moura (2012) Mixed-mode I+II continuum damage model applied to fracture characterization of bonded joints. Journal of Adhesion and Adhesives, 41, 92-97. 